\title{
Calculations of $B_{1}$ Distribution, Specific Energy Absorption Rate, and Intrinsic Signal-to-Noise Ratio for a Body-Size Birdcage Coil Loaded with Different Human Subjects at 64 and $128 \mathrm{MHz}$
}

\author{
W. Liu ${ }^{1}$, C. M. Collins ${ }^{1}$, and M. B. Smith ${ }^{1,2}$ \\ ${ }^{1}$ Department of Radiology, Pennsylvania State University College of Medicine, Hershey, \\ Pennsylvania, USA \\ ${ }^{2}$ Department of Cellular and Molecular Physiology, Pennsylvania State University College of \\ Medicine, Hershey, Pennsylvania, USA
}

\section{Abstract}

\begin{abstract}
A numerical model of a female body is developed to study the effects of different body types with different coil drive methods on radio-frequency magnetic $\left(\mathbf{B}_{1}\right)$ field distribution, specific energy absorption rate (SAR), and intrinsic signal-to-noise ratio (ISNR) for a body-size birdcage coil at 64 and $128 \mathrm{MHz}$. The coil is loaded with either a larger, more muscular male body model (subject 1) or a newly developed female body model (subject 2), and driven with two-port (quadrature), four-port, or many (ideal) sources. Loading the coil with subject 1 results in significantly less homogeneous $\mathbf{B}_{1}$ field, higher SAR, and lower ISNR than those for subject 2 at both frequencies. This dependence of MR performance and safety measures on body type indicates a need for a variety of numerical models representative of a diverse population for future calculations. The different drive methods result in similar $\mathbf{B}_{1}$ field patterns, SAR, and ISNR in all cases.
\end{abstract}

\section{Introduction}

\begin{abstract}
Estimating radio-frequency (RF) field distribution, RF power deposition and specific absorption rate (SAR), and the signal-to-noise ratio (SNR) in the whole human body has been an interest in magnetic resonance imaging (MRI) for decades along with the trend of MRI systems toward higher fields. Experimental measurements have been performed accompanied by simulations with homogeneous models. Bottomley et al. [1, 2] calculated the RF power deposition and SAR in body-size muscle phantoms exposed to homogeneous fields up to $100 \mathrm{MHz}$ and performed experimental measurements on subjects at $63 \mathrm{MHz}$. Hoult and Lauterbur [3] and Chen et al. [4] developed methods to calculate the loss and SNR in the human subject. Glover et al. [5] compared the power deposition and SNR in a lossy body-size phantom and in human bodies for linear and quadrature excitation at 63 MHz. Edelstein et al. [6] introduced the concept of intrinsic SNR (ISNR) to MR human imaging. Carlson [7] proposed a general solution for correlation between power deposition and noise using long wave-length and large skin depth approximations. More complex body models were developed in the 90s. Grandolfo et al. [8] calculated SAR in a 13-tissue humantorso model at up to $64 \mathrm{MHz}$ with the impedance method. Wen et al. [9] measured the power deposition and ISNR in heart imaging at 1.5, 3, and $4 \mathrm{~T}$ and Singerman et al. [10] simulated the results with a multilayer axis-symmetric chest model by finite element methods. Gandhi
\end{abstract}


and Chen [11] calculated SAR in a 30-tissue body model, whose anatomy was based on MRI scan images of a male, from 64 to $350 \mathrm{MHz}$ with the finite difference time domain (FDTD) method [12, 13]. Collins and Smith [14] also developed an anatomically accurate body model for FDTD by segmenting photographic image slices of a male cadaver and presented calculations of $\mathbf{B}_{1}$ field, SAR, and SNR matching experiments of Wen et al. [9] well. Interestingly, most subjects, if not all, in the experiments [2, 5, 9] or simulations [11, 14] were males. No direct comparison of $\mathbf{B}_{1}$ field distribution, RF power deposition, or SNR between any two different bodies has been performed previously in calculations.

Here we develop another anatomically accurate body model based on an individual female body, which is quite different from the existing male body model in size, shape, and composition. We chose to create a second model based on a second individual because merely re-scaling an existing body model could produce unrealistic, disproportionate possibilities and so that more options would be available for future studies requiring body models. We directly compare the $\mathbf{B}_{1}$ field distribution, SAR, and SNR in the male model (subject 1) developed by Collins et al. [14] with those in the newly developed female model (subject 2) using the FDTD method. Both models were loaded into a body-size twenty-fourrung highpass birdcage coil [15] at 64 and $128 \mathrm{MHz}$. The three drive methods include conventional quadrature excitation [5], four-port excitation [16, 17], and ideal excitation [18, 19], which assumes the current distribution in the coil rungs varies sinusoidally with angle in the azimuthal plane.

\section{Methods}

A twenty-four-rung body-size (diameter, $63 \mathrm{~cm}$; shield diameter, $68 \mathrm{~cm}$; length, $70 \mathrm{~cm}$ ) shielded highpass birdcage coil loaded with two different subjects was modeled at 64 and $128 \mathrm{MHz}$ using the FDTD method (Fig. 1). Each rung was modeled as a $5 \mathrm{~cm}$ wide copper strip and each end-ring was $10 \mathrm{~cm}$ wide. Subject 1 was a male with approximate height of $180.3 \mathrm{~cm}$ and mass of $90.3 \mathrm{~kg}$. Subject 2 was a female with approximate height of $165.1 \mathrm{~cm}$ and unknown mass. We created the FDTD model of subject 1 by modifying the model used in previous calculations [14]: the thickness of skin was decreased to be more realistic. The model of subject 2 was newly created by segmenting the images of the female cadaver from the National Libraries of Medicine's Visible Human Project and then assigning appropriate electric conductivity, electric permittivity, and mass density to each tissue at each frequency [14, 20-24], The values of these properties for all the tissue types (33 for the male and 36 for the female) are listed in Table 1. The resolution of the final FDTD meshes is $5 \mathrm{~mm}$ in all three dimensions, which has been shown to be adequate for SAR calculations in a head model at $64 \mathrm{MHz}$ [25]. The heights of the models are the same as the subjects; however, the mass of subject 1 calculated from the model is about $108.9 \mathrm{~kg}, 20.6 \%$ more than the subject's actual mass. The mass calculated from the model of subject 2 is about $85.5 \mathrm{~kg}$. Although the actual mass of subject 2 is not available, we would expect that the mass calculated from this model is also larger than the subject's actual mass to about the same degree as that for subject 1 since the procedures of making the models are identical. One major cause of the mass difference between the model and the subject is that the volume of cadavers expanded during freezing so the model is bigger, but not taller, than the subject. So we actually modeled a subject that has a similar body structure but a bigger size than the original subject. Other causes include the extra skin tissue added in the model to ensure the continuity of skin [14] and the limited resolution of the model. Subject 1 has more muscle tissue ( $38 \%$ of the body mass) than subject 2 ( $24 \%$ of the body mass).

Each subject was loaded into the coil model so that the center of the heart was on the center axial plane of the coil and the back of the subject (or patient bed) was approximately $10 \mathrm{~cm}$ 
from the axis of the coil. This way the widest portion of the subjects in the left-right direction coincided with the widest portion of the coil.

Each coil was tuned to each desired frequency when loaded with each subject with a method using ideal sources, determining the corresponding average impedance across each capacitor, and then calculating the capacitance needed to provide that impedance [26]. The coils were then driven in conventional quadrature (two-port), four-port, and ideal configurations. Two equal amplitude voltage sources were placed in the superior end-ring $90^{\circ}$ apart in space and in phase $\left(0\right.$ and $\left.90^{\circ}\right)$ for the conventional quadrature drive. For the four-port excitation, four equal amplitude voltage sources with a $90^{\circ}$ phase shift between each adjacent pair $\left(0,90,180\right.$, and $\left.270^{\circ}\right)$ were placed in the superior end-ring (Fig. 2). Fortyeight capacitors in the two end-rings were modeled to tune the coil to the desired resonant frequencies. For the ideal excitation, forty-eight sources placed in the two end-rings were used to perform the function of the capacitors [19]. They had the same amplitude but their phases were equal to the azimuthal angles. A voltage source in the inferior end-ring had a phase $180^{\circ}$ shifted from the phase of the voltage source at the same location in the superior end-ring. These voltage sources were defined such that they would result in an ideal current distribution for a homogeneous $\mathbf{B}_{1}$ field if the coil were unloaded. This method has provided calculated $\mathbf{B}_{1}$ distributions in good agreement with experimentally measured distributions at frequencies up to $128 \mathrm{MHz}$ for a head-size birdcage coil [27]. All FDTD calculations were set up and solved with the aid of commercially available software (xFDTD, Remcom; State College, PA). The calculation space was $2.19 \mathrm{~m}$ in the inferior-superior direction and 1.11 $\mathrm{m}$ in both the left-right and anterior-posterior directions. A second-order Liao absorbing boundary condition [28] was used at all boundaries of the problem region. There were at least twenty cells between the outer boundary and any coil or body structures. Each calculation was run for twenty-four cycles to ensure the steady state was reached. The magnitudes of $\mathbf{B}_{1}$ fields and $\mathbf{E}$ fields were scaled as to maximize the amplitude of the total signal on the central axial plane contributing to a reconstructed gradient echo image with a 3 $\mathrm{ms} 90^{\circ}$ rectangular RF pulse and a long TR [29].

$B_{1}^{+}$, the magnitude of the component of the $\mathbf{B}_{1}$ field that rotates in the same direction of the nuclear precession, and, $B_{1}^{-}$, the magnitude of the component that rotates in the opposite direction of the nuclear precession, were calculated from the results of two separate field calculations [30]

$$
B_{1}^{+}=\left|\frac{\left(\widehat{B}_{x}+\mathrm{i} \widehat{B}_{y}\right)}{2}\right|, B_{1}^{-}=\left|\frac{\left(\widehat{B}_{x}-\mathrm{i} \widehat{B}_{y}\right)^{*}}{2}\right|,
$$

where the hat accent indicates the complex amplitude [29], $\mathrm{i}$ is the imaginary unit, and the asterisk indicates the complex conjugate. The phases of the voltage sources in the field calculation for $B_{1}^{+}$are opposite those in the field calculation for $B_{1}^{-}$[29].

The flip angle of the $n$th Yee cellshift $a_{n}$ on the central axial plane was calculated as [29]

$$
\alpha_{n}=B_{1 n}^{+} \gamma \tau,
$$

where $B_{1 n}^{+}$is $B_{1}^{+}$of the $n$th Yee cell, $\gamma$ is the gyromagnetic ratio of ${ }^{l} \mathrm{H}$ and $\tau$ is the duration of the RF pulse. In this study, $\tau$ is equal to $3 \mathrm{~ms}$. The average flip angle on the whole plane, which would be $90^{\circ}$ in a perfectly homogeneous $B_{1}^{+}$field, was calculated by dividing the 
summation of the flip angles on the central plane by the total number of Yee cells in the body model on that plane.

The ISNR was calculated on the central axial plane of the coil, which is through the heart of the subject, with the formula [29]

$$
\operatorname{ISNR} \propto \frac{f^{2}}{N_{\text {plane }} \sqrt{P_{\text {abs }}}} \sum_{N_{\text {plane }}}\left|W_{n}\left(\operatorname{Sin} \alpha_{n}\right) B_{1 n}^{-}\right|,
$$

where $f$ is the Larmor frequency, $N_{\text {plane }}$ is the number of the Yee cells in the body model on the center axial plane, $W_{n}$ is the water content [31] of the $n$th Yee cell, $a_{n}$ is the flip angle of the $n$th Yee cell, $B_{1 n}^{-}$is $B_{1}^{-}$of the $n$th Yee cell, and $P_{\text {abs }}$, the total absorbed power in the entire model, was calculated as [29]

$$
P_{\mathrm{abs}}=\frac{1}{2} \sum_{N_{\mathrm{body}}}\left(\sigma_{x n} E_{x n}^{2}+\sigma_{y n} E_{y n}^{2}+\sigma_{z n} E_{z n}^{2}\right) \Delta_{x} \Delta_{y} \Delta_{z},
$$

where $N_{\text {body }}$ is the total number of Yee cells in the whole model, $\sigma$ is the electric conductivity, subscript $n$ indicates the $n$th Yee cell, $E$ is the magnitude of electric field intensity, $\Delta_{X}, \Delta_{y}$, and $\Delta_{Z}$ are the dimensions of the Yee cell in three directions. This method of calculating ISNR has been shown to be in good agreement with experiment in comparison of SNR in the human head at different field strengths [32]

In concept, SAR can be calculated as

$$
\mathrm{SAR}=\frac{\sigma}{2 \rho} E^{2}
$$

where $\rho$ is the mass density of the tissue. There are many possible approaches for calculating SAR over masses or volumes consisting of multiple Yee cells. These methods can be quite involved and discussion of them is beyond the scope of this work. For the calculation of the local maximum SAR in one gram of tissue $\left(S_{A} R_{L}\right)$, we employed a widely used method that uses twelve electrical field components to calculate SAR in each cell, and then includes cells about a central cell in a strategic order until a mass of interest is reached [33]. The SAR averaged over the entire body model $\left(\mathrm{SAR}_{\mathrm{W}}\right)$ was calculated by first calculating the total power absorbed $\left(P_{\mathrm{abs}}\right)$ in the subject and then dividing by the total subject mass, similar to how it would be done in experiment. All SAR values are presented as for a $100 \%$ duty cycle.

\section{Results}

The distributions of $B_{1}^{+}$on the central axial plane in the empty coil and in the coil loaded with subject 1 at $128 \mathrm{MHz}$ with the conventional quadrature excitation, the four-port excitation, and the ideal excitation are shown in Fig. 3. The $B_{1}^{+}$fields are homogeneous in the empty coils, with about $98 \%$ of the area within a diameter of $0.56 \mathrm{~m}(90 \%$ of the coil diameter) on the plane shown having a homogeneity in the empty coil, calculated by $\mid B_{1}^{+}(r)-B_{1}^{+}$average $|/| B_{1}^{+}$average $\mid$within $10 \%$ for all three excitations. These results indicate the coils are resonant at the correct mode for MRI. Loading the coil with the subject, however, 
distorts the field distribution dramatically, so that only about $30 \%$ of the area in the body on the plane shown has homogeneity within $10 \%$.

The average flip angle in the subjects on the central axial plane when the coil is driven as if to maximize the integrated signal on a gradient echo image with long TR is shown in Table 2. Also shown is the homogeneity of the flip angle distribution, which is defined as the percentage of the total area that has flip angles within $\pm 30 \%$ deviation of the average flip angle on the central axial plane. The larger the number, the more homogeneous the field. The $P_{\mathrm{abs}}, \mathrm{SAR}_{\mathrm{W}}, \mathrm{SAR}_{\mathrm{L}}$, and relative ISNR on the central axial plane are also shown in Table 2. ISNR values are normalized such that the ISNR in subject 1 with quadrature excitation at $64 \mathrm{MHz}$ is 1 .

Figure 4 contains plots of the flip angle distribution on the central axial plane for the fourport excitation in both subjects at both frequencies. Figure 5 shows the SAR distribution in the sagittal, coronal, and axial planes through the location where $\mathrm{SAR}_{\mathrm{L}}$ occurs with the fourport excitation at $128 \mathrm{MHz}$.

\section{Discussion}

\subsection{Three Drive Methods}

With the exception of $\mathrm{SAR}_{\mathrm{L}}$, all three drive methods produce results within $4.5 \%$ of each other at $64 \mathrm{MHz}$ and within $7.2 \%$ of each other at $128 \mathrm{MHz}$ (Table 2). This indicates that when the coil is on resonance, either 4-port or quadrature drives are capable of producing a field distribution much like that in an ideal birdcage coil. It is important to note, however, that in these models all capacitor values are identical. The presence of tuning, matching, and/or decoupling capacitors within the coil structure can produce significant perturbations in current and field distributions [34]. Further calculations with the coil rotated $7.5^{\circ}$ so that rungs were centered in the gaps between rungs in the current orientation resulted in differences of less than $1 \%$ in any results even at $128 \mathrm{MHz}$, indicating that for 24-rung coils, field distributions are very in-dependent of coil orientation. The values for $\mathrm{SAR}_{\mathrm{L}}$ are very sensitive to small changes in field perturbations, which can result in changes in location as well as in magnitude of $\mathrm{SAR}_{\mathrm{L}}$. The largest difference of $\mathrm{SAR}_{\mathrm{L}}$ depending on drive method is about $13 \%$ at $64 \mathrm{MHz}$ and about $15 \%$ at $128 \mathrm{MHz}$, both in subject 1 . Considering the variation of local SAR throughout the sample, these differences are relatively small.

Unlike the conventional quadrature excitation and the four-port excitation, which are used in experiments [16], the ideal excitation only exists in numerical modeling. With the ideal excitation, the current in each rung of the coil when empty follows the pattern that would produce a homogeneous RF field in the empty coil, and the interaction between the sample and the loaded coil is then ignored. This method requires less computation time by skipping the fine tuning of the RF coil and has been given results similar to experiment in a head model within a birdcage coil up to $128 \mathrm{MHz}$ [27]. The similarity between results with different drive methods in this study indicates that the ideal excitation can also provide an accurate prediction of fields in quadrature and four-port coils at up to $128 \mathrm{MHz}$ for a body coil loaded with body models.

\section{$4.2 B_{1}^{+}$Field Distribution}

The plots of the flip angle distribution on the central axial plane for the four-port excitation are shown in Fig. 4. The flip angle distribution plots for the conventional quadrature and ideal excitations are similar to those of four-port excitations and are not shown here. 
In all cases, the distribution of the flip angles is more homogeneous at $64 \mathrm{MHz}$ than that at $128 \mathrm{MHz}$ as expected. At $128 \mathrm{MHz}$, a black band, indicating an area that has smaller flip angles, is observed in the back muscles in the subject along with bright regions, indicating areas that have larger flip angles, at the edges of the arms (Fig. 4). The average flip angle ranges more narrowly at $64 \mathrm{MHz}$ (from 88.67 to $89.00^{\circ}$ ) than at $128 \mathrm{MHz}$ (from 73.26 to $84.45^{\circ}$ ) with different subjects and different drive methods. The homogeneity ranges from 98.25 to $99.90 \%$ at $64 \mathrm{MHz}$ and from 72.65 to $83.33 \%$ at $128 \mathrm{MHz}$.

The distributions of the flip angles in subject 1 is always less homogeneous than that in subject 2 . This is because subject 1 has a larger chest size than subject 2 and because subject 1 contains more muscle, which has stronger dielectric properties than does fat. Averaged between driving methods, the difference in homogeneity between subject 1 and subject 2 is about $2 \%$ at $64 \mathrm{MHz}$ and about $9.6 \%$ at $128 \mathrm{MHz}$.

The $B_{1}^{+}$field distributions produced by the different drive methods are very similar. In all cases the difference is within $7.2 \%$.

\subsection{SAR}

Averaged among different coil excitation methods, the $\mathrm{SAR}_{\mathrm{W}}$ in subject 1 is about $0.59 \mathrm{~W} /$ $\mathrm{kg}$ at $64 \mathrm{MHz}$ and $1.54 \mathrm{~W} / \mathrm{kg}$ at $128 \mathrm{MHz}$. The $\mathrm{SAR}_{\mathrm{W}}$ in subject 2 is about $0.46 \mathrm{~W} / \mathrm{kg}$ at 64 $\mathrm{MHz}$ and $1.37 \mathrm{~W} / \mathrm{kg}$ at $128 \mathrm{MHz}$, about $22 \%$ and $11 \%$ less than that in subject 1 at corresponding frequencies. Considering the difference of body mass, flip angle, pulse duration, coil, dimension, and coil drive method, the results at $64 \mathrm{MHz}$ are in good agreement with experiments [2]. Local SAR levels are highest in skin and muscle tissues near the periphery of the body in both subjects. At both frequencies, the $\mathrm{SAR}_{\mathrm{L}}$ in subject 1 is always located at the skin and muscle tissues near the right clavicle (Fig. 5). In subject 2, the $\mathrm{SAR}_{\mathrm{L}}$ occurs in the triceps of the right arm at $64 \mathrm{MHz}$ and in the triceps of the left arm at $128 \mathrm{MHz}$ (Fig. 5). Since both subjects' hands are in contact with the body, these locations may be in the path of a large eddy current loop that includes the torso and the arms [35]. Averaged among different coil drive methods, the $\mathrm{SAR}_{\mathrm{L}}$ in subject 1 is $9.97 \mathrm{~W} / \mathrm{kg}$ at 64 $\mathrm{MHz}$ and $26.8 \mathrm{~W} / \mathrm{kg}$ at $128 \mathrm{MHz}$. The $\mathrm{SAR}_{\mathrm{L}}$ in subject 2 is $6.56 \mathrm{~W} / \mathrm{kg}$ at $64 \mathrm{MHz}$ and 19.1 $\mathrm{W} / \mathrm{kg}$ at $128 \mathrm{MHz}$, about $34.2 \%$ lower than that in subject 1 at $64 \mathrm{MHz}$ and about $28.6 \%$ lower at $128 \mathrm{MHz}$. Both $\mathrm{SAR}_{\mathrm{W}}$ and $\mathrm{SAR}_{\mathrm{L}}$ are calculated for a $100 \%$ duty cycle here. They can be easily converted to a format that can be directly compared with the IEC regulations [36] for different imaging sequences [18].

No notable difference (less than $4.5 \%$ ) in $\mathrm{SAR}_{\mathrm{W}}$ between the drive methods is observed at either frequency. The difference in $\mathrm{SAR}_{\mathrm{L}}$ between excitation methods is below $15 \%$ in subject 1 and below $8.9 \%$ in subject 2 at both frequencies.

\subsection{ISNR}

When using the body coil both for transmitting and receiving, the RF power deposited in the body can also be used to estimate the noise in ISNR calculations [6,7]. The power absorbed is from 63.64 to $64.54 \mathrm{~W}$ in subject 1 and from 38.81 to $40.03 \mathrm{~W}$ in subject 2 with different drive methods at $64 \mathrm{MHz}$. These results are also in good agreement with previous experiments [2] considering the difference of flip angle, pulse duration, coil dimensions, and coil drive method. At $128 \mathrm{MHz}$, the power absorbed is from 165.2 to $168.4 \mathrm{~W}$ in subject 1 and from 114.7 to $118.8 \mathrm{~W}$ in subject 2 with different drive methods. The absorbed power increases with the frequency as expected theoretically. The rate of the increase, however, is less than the theoretical value $f^{2}$. The power deposition averaged between excitation methods at $128 \mathrm{MHz}$ is about $34.7 \%$ less than the theoretically expected value in subject 1 and about $25 \%$ less for subject 2 . This less than quadratic increase has been observed in a 
head model at frequencies above $175 \mathrm{MHz}$ [29]. It occurs here at a frequency as low as 128 $\mathrm{MHz}$ because the large size of the body makes the phase variation of $\mathbf{B}_{1}$ on any transecting plane significant enough to cause cancellation of the net magnetic flux through the plane resulting in less absorbed power according to Faraday's law [29]. This less than quadratic increase in $P_{\text {abs }}$ could be used to explain the greater than linear increase in ISNR with frequency (about $4.2 \%$ greater SNR at $128 \mathrm{MHz}$ than that predicted linearly) in subject 2. In subject 1 , the much less homogeneous flip angle distribution may diminish this effect of lower power deposition on ISNR, resulting in an ISNR about 3.3\% less than a prediction assuming a linear increase with $\mathbf{B}_{0}$.

Loading subject 2 results in higher ISNR than that of loading subject 1 in all cases. The difference is about $12 \%$ at $64 \mathrm{MHz}$ and $20.6 \%$ at $128 \mathrm{MHz}$ averaged between different drive methods. Smaller body size, less muscle tissue, better RF penetration, and more-

homogeneous $\mathbf{B}_{1}$ field distribution are among the contributors for the higher ISNR in subject 2.

\section{Conclusions}

Measures of MR performance and safety are very dependent on the body type of the subject. Loading a larger, more muscular subject results in significantly less homogeneous $B_{1}^{+}$ distribution, higher RF power deposition, lower ISNR, and higher SAR levels at both 64 and $128 \mathrm{MHz}$. All drive methods result in similar $B_{1}^{+}$field patterns, SAR levels, and ISNR. Compared to the conventional quadrature excitation and the four-port excitation, the ideal excitation is very accurate at $64 \mathrm{MHz}$ and in most cases at $128 \mathrm{MHz}$ for these body-size coil simulations. The effects of sample position in the coil and the presence of tuning and matching capacitors (rather than equal-value capacitors throughout coil) may be significant, but were not investigated in this study. The dependence of MR performance and safety measures on body type indicates a need for a variety of numerical models representative of a diverse population for future calculations.

\section{Acknowledgments}

We are grateful to the National Institutes of Health for funding of this project through R01 EB000454.

\section{References}

1. Bottomley PA, Andrew E. Phys. Med. Biol. 1978; 23:630-643. [PubMed: 704667]

2. Bottomley PA, Redington RW, Edelstein WA, Schenck JF. Magn. Reson. Med. 1985; 2:336-349. [PubMed: 4094551]

3. Hoult D, Lauterbur P. J. Magn. Reson. 1979; 34:425-433.

4. Chen C-N, Sank V, Cohen S, Hoult D. Magn. Reson. Med. 1986; 3:722-729. [PubMed: 3784889]

5. Glover GH, Hayes CE, Pelc NJ, Edelstein WA, Mueller OM, Hart HR, Hardy CJ, O’Donnell M, Barber WD. J. Magn. Reson. 1985; 64:255-270.

6. Edelstein W, Glover GH, Hardy C, Redington RW. Magn. Reson. Med. 1986; 3:604-618. [PubMed: 3747821]

7. Carlson J. Magn. Reson. Med. 1989; 10:399-403. [PubMed: 2733595]

8. Grandolfo M, Vecchia P, Gandhi O. Bioelectromagnetics. 1990; 11:117-128. [PubMed: 2242047]

9. Wen H, Denison T, Singerman R, Balaban R. J. Magn. Reson. 1997; 125:65-71. [PubMed: 9245361]

10. Singerman RW, Denison TJ, Wen H, Balaban RS. J. Magn. Reson. 1997; 125:72-83. [PubMed: 9245362]

11. Gandhi O, Chen X. Magn. Reson. Med. 1999; 41:816-823. [PubMed: 10332859] 
12. Yee KS. IEEE Trans. Antennas Propag. 1966; 14:302-307.

13. Kunz, KS.; Luebbers, RJ. The Finite Difference Time Domain Method for Electromagnetics. Ann Arbor: CRC; 1993.

14. Collins CM, Smith MB. Magn. Reson. Med. 2001; 45:692-699. [PubMed: 11283998]

15. Hayes CE, Edelstein WA, Schenck JF, Mueller OM, Eash M. J. Magn. Reson. 1985; 63:622-628.

16. Ledden, PJ.; Wald, LL.; Vaughan, JT. In: Moonen, CT., editor. Proceedings of the Eighth Scientific Meeting of the International Society for Magnetic Resonance in Medicine; April 1-7, 2000; Denver, Colorado, USA. Denver, CO: ISMRM; 2000. p. 1395

17. Ibrahim TS, Lee R, Baertlein BA, Kangarlu A, Robitaille PML. Magn. Reson. Imaging. 2000; 18:733-742. [PubMed: 10930783]

18. Collins CM, Li S, Smith MB. Magn. Reson. Med. 1998; 40:847-856. [PubMed: 9840829]

19. Collins, CM. Calculations of RF Magnetic Fields and SAR Experienced by the Human Body during MRI. Philadelphia, Pa: University of Pennsylvania; 1999. p. 200

20. Gabriel, C. Compilation of the Dielectric Properties of Body Tissues at RF and Microwave Frequencies. Brooks Air Force Base, Texas: Air Force Material Command; 1996. Report AL/OETR-1996-0037

21. Erdmann WS, Gos T. J. Biomech. 1990; 23:945-947. [PubMed: 2211740]

22. Huang HK, Wu SC. Comput. Biol. Med. 1976; 6:337-343. [PubMed: 1000959]

23. Cho ZH, Tsai CM, Wilson G. Phys. Med. Biol. 1975; 20:879-889. [PubMed: 1202507]

24. Clauser, CE.; McConville, JT.; Young, JW. Weight, Volume, and Center of Mass of Segments the Human Body. Wright-Patterson Air Force Base, Ohio: Aerospace Medical Research Laboratory; 1969. Report AMRL-TR-69-70

25. Collins CM, Smith MB. J. Magn. Reson. Imaging. 2003; 18:383-388. [PubMed: 12938138]

26. McKinnon, G.; Wang, Z. In: Lom, DJ., editor. Proceedings of the 11th Scientific Meeting of the International Society for Magnetic Resonance in Medicine; July 10-16, 2003; Toronto, Ontario, Canada. Toronto, Ontario: ISMRM; 2003. p. 2381

27. Alecci M, Collins CM, Smith MB, Jezzard P. Magn. Reson. Med. 2001; 46:379-385. [PubMed: $11477643]$

28. Liao ZP, Wong HL, Yang GP, Yuan YF. Sci. Sin. A. 1984; 27:1063-1076.

29. Collins CM, Smith MB. Magn. Reson. Med. 2001; 45:684-691. [PubMed: 11283997]

30. Hoult DI. Concepts Magn. Reson. 2000; 12:173-187.

31. Duck, FA. Physical Properties of Tissue - A Comprehensive Reference Book. New York: Academic Press; 1990.

32. Vaughan JT, Garwood M, Collins CM, Liu W, DelaBarre L, Adriany G, Andersen P, Merk H, Goebel R, Smith MB, Uğurbil K. Magn. Reson. Med. 2001; 46:24-30. [PubMed: 11443707]

33. Caputa K, Okeniewski M, Stuchly MA. IEEE Antennas Propag. Mag. 1999; 41:102-107.

34. Jin J, Shen G, Perkins T. Magn. Reson. Med. 1994; 32:418-422. [PubMed: 7984076]

35. Zhai, Z.; DeMeester, GD.; Shvartsman, SM.; Morich, MA. In: Evelhoch, JZ., editor. Proceedings of the 10th Scientific Meeting of the International Society for Magnetic Resonance in Medicine; May 18-24, 2002; Honolulu, Hawaii, USA. Honolulu, Hawaii, USA: ISMRM; 2002. p. 773

36. International Electrotechnical Commission: Medical electrical equipment part 2-33: Particular requirements for the safety of magnetic resonance equipment for medical diagnosis. Reference IEC 60601 1-2-33:2002(E). Geneva: International Electrotechnical Commission; 2002. 


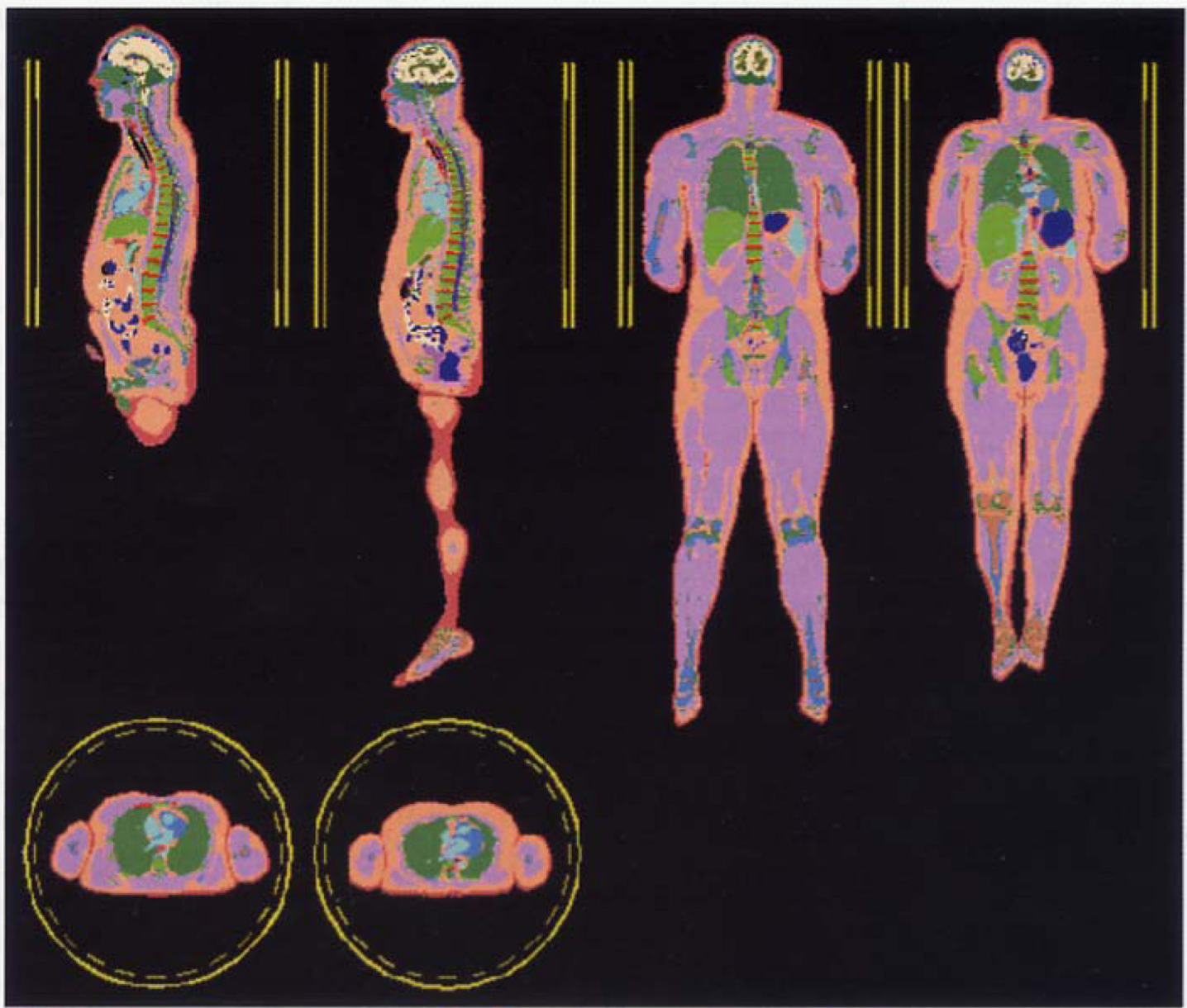

\begin{tabular}{|l|l|l|}
\hline Skin & Nerve & Pancreas \\
\hline Tendon and undefined & Cartilage & Gall bladder \\
\hline Fat & Bowel content & Kidney \\
Cortical bone & Adrenal gland & Bladder \\
\hline Cancellous bone & Thyroid & Testicles, Mammary tissue \\
Blood & Stomach & Cerebellum \\
Muscle & Lung & Yellow marrow \\
Grey matter & Aorta & Bladder content \\
White matter & Heart & Bile, Great vestibular gland \\
Cerebro-spinal fluid & Liver & Uterus content \\
Sclera, Cornea & Spleen & Uterus, vagina \\
Vitreous humour & Colon & Tongue \\
\hline Lens & Intestine & Copper \\
\hline
\end{tabular}

Fig. 1.

Sagittal, coronal, and axial cross sections through models of subject 1 (left) and subject 2 (right) through the center of the coil. 


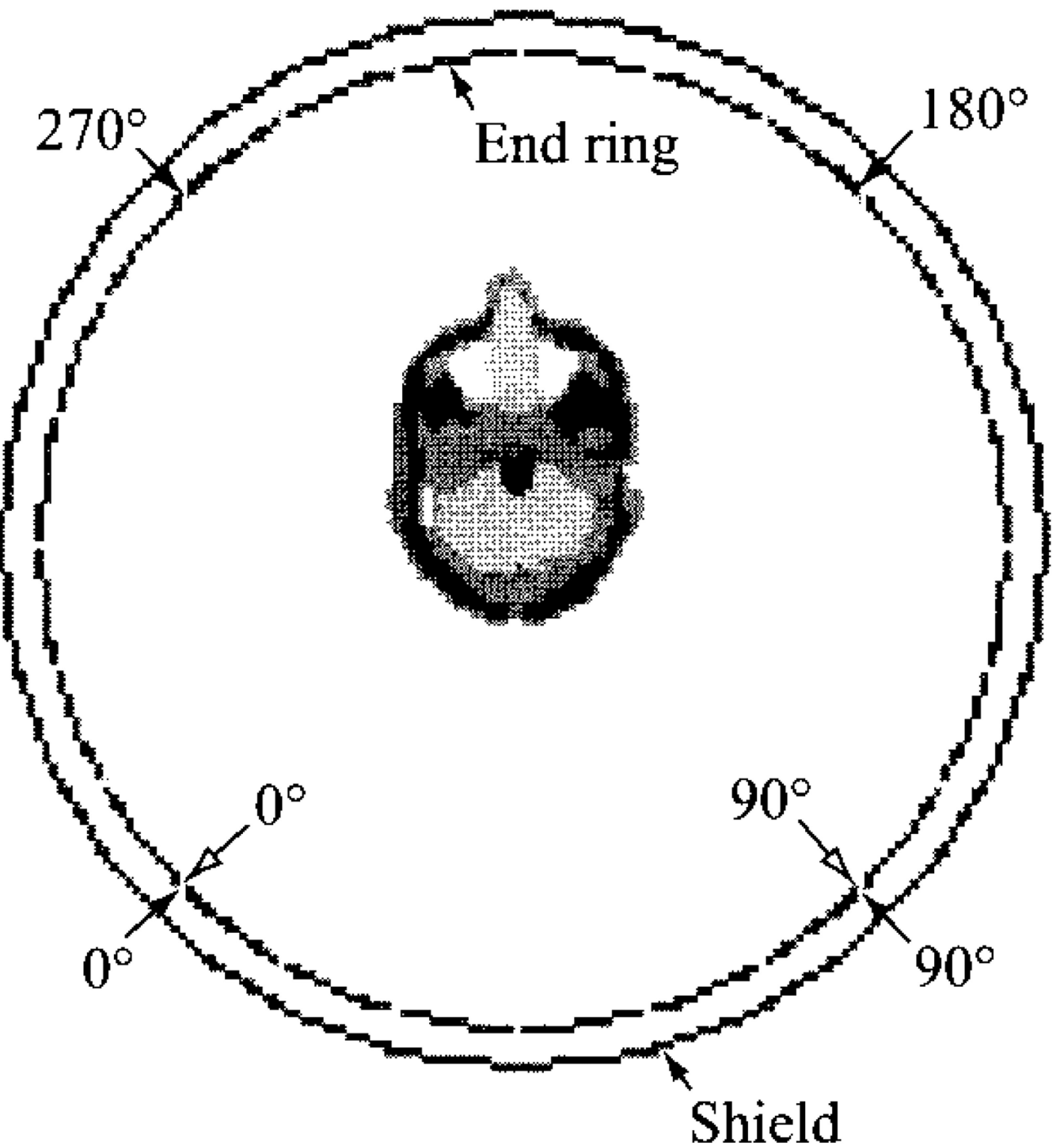

$\searrow$ Port for conventional quadrature excitation Port for four-port excitation

Fig. 2.

Location of the drive ports in the conventional quadrature excitation and in the four-port excitation. 


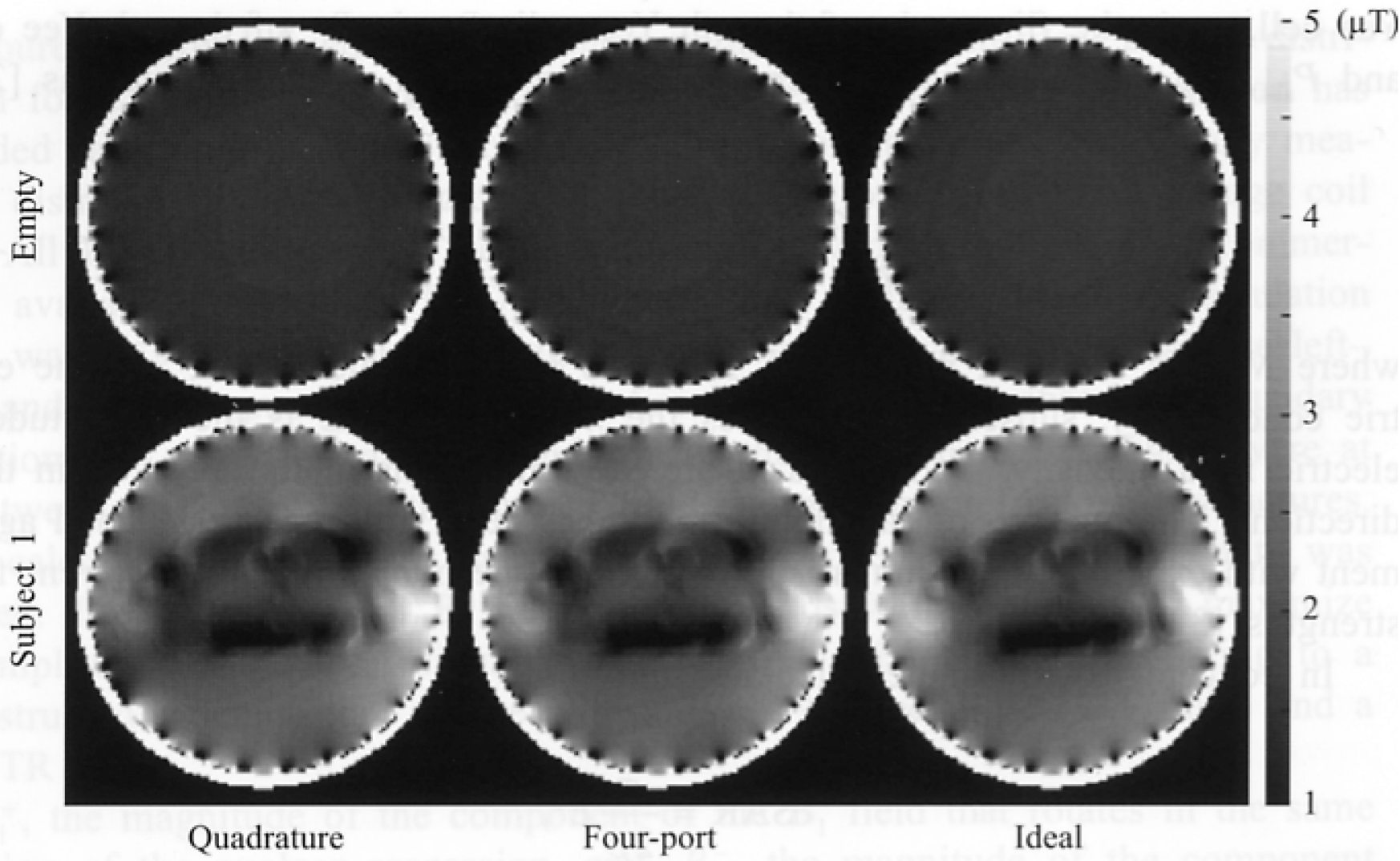

Fig. 3.

Distribution of $B_{1}^{+}$on the central axial plane in the empty coils and in the coils loaded with subject 1 for the quadrature excitation, the four-port excitation, and the ideal excitation at $128 \mathrm{MHz} . B_{1}^{+}$at the center of the coil is normalized to $1.96 \mu \mathrm{T}$, the field strength necessary to produce a $3 \mathrm{~ms} 90^{\circ}$ rectangular RF pulse. $B_{1}^{+}$values above $8 \mu \mathrm{T}$ appear as $5 \mu \mathrm{T}$. 


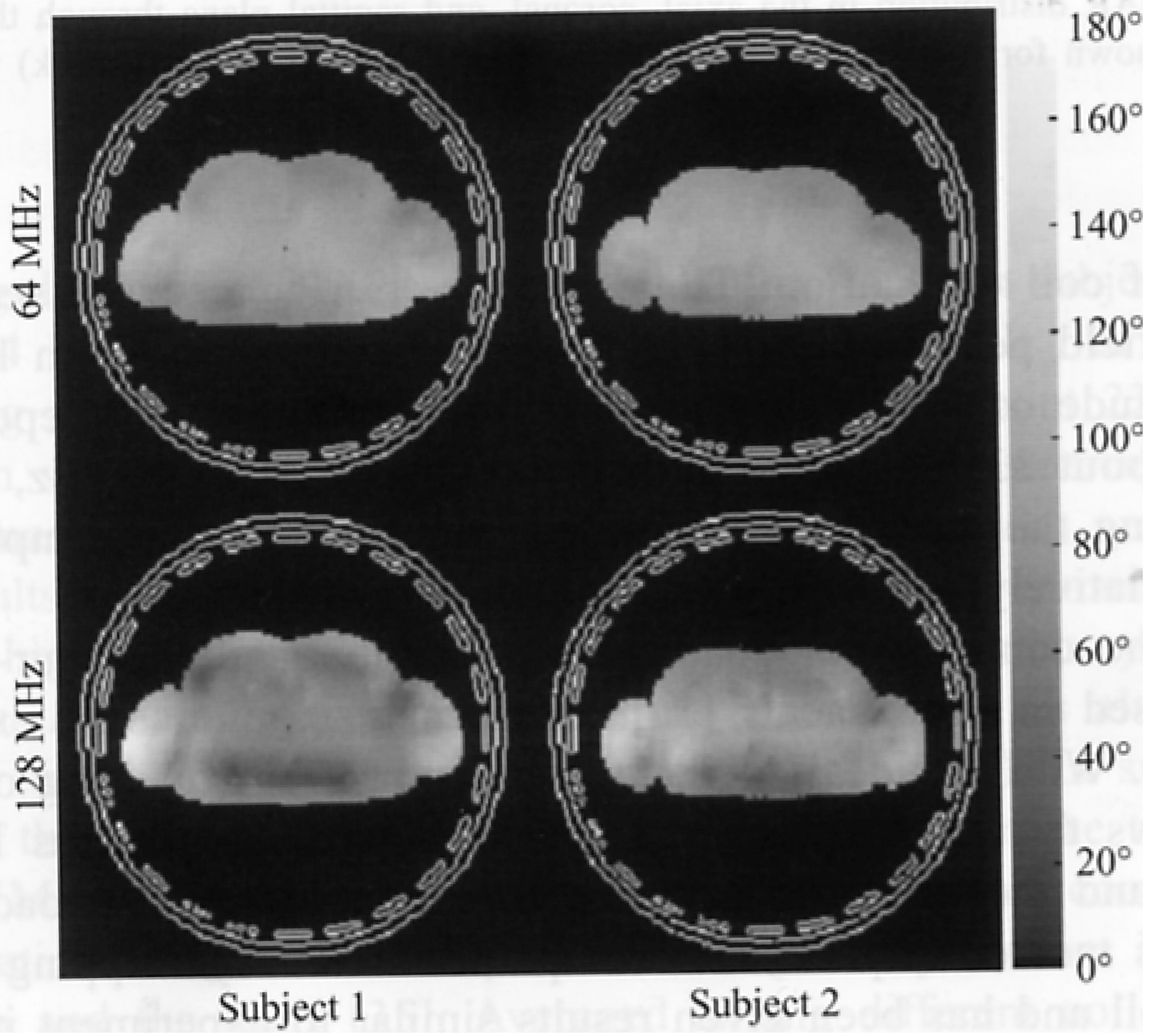

Fig. 4.

Distribution of flip angles for maximum signal on a reconstructed gradient echo image with a $3 \mathrm{~ms}$ rectangular RF pulse and long TR on the central axial plane shown for the four-port excitation. 


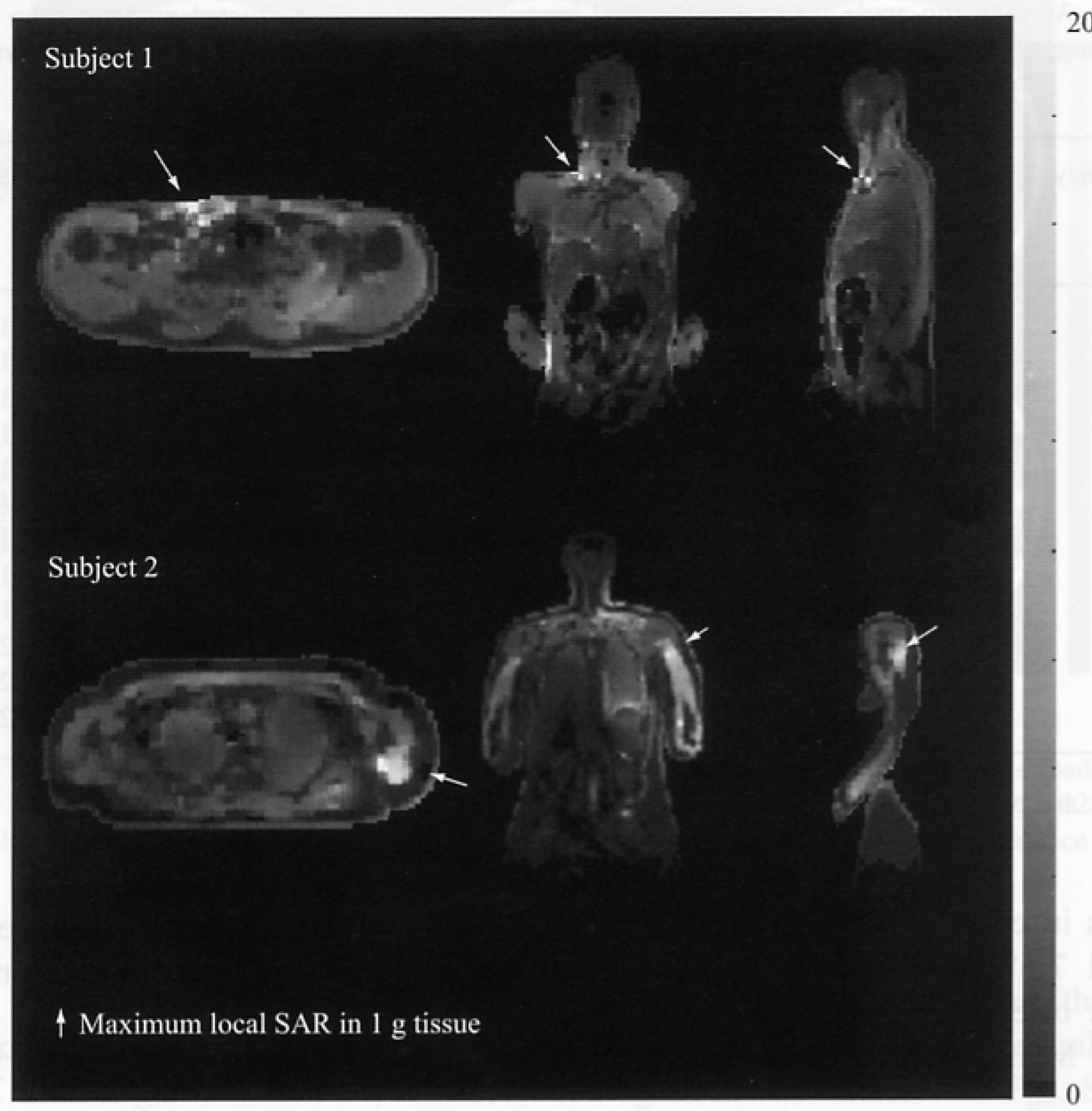

Fig. 5.

Local SAR distribution in the axial, coronal, and sagittal plane through the location of $\mathrm{SAR}_{\mathrm{L}}$ at $128 \mathrm{MHz}$, shown for four-port excitation. Linear gray scale is from 0 (black) to $20 \mathrm{~W} / \mathrm{kg}$ (white).Values above $20 \mathrm{~W} / \mathrm{kg}$ appear as $20 \mathrm{~W} / \mathrm{kg}$. 


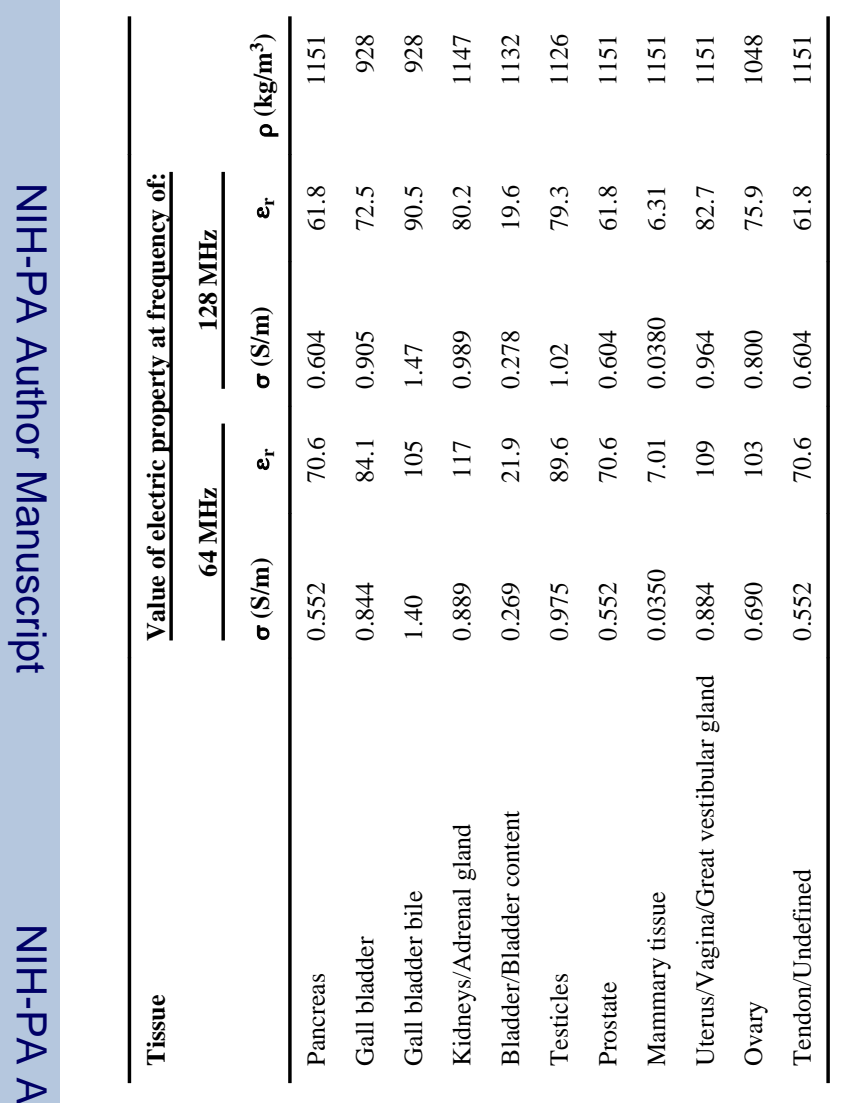

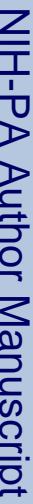




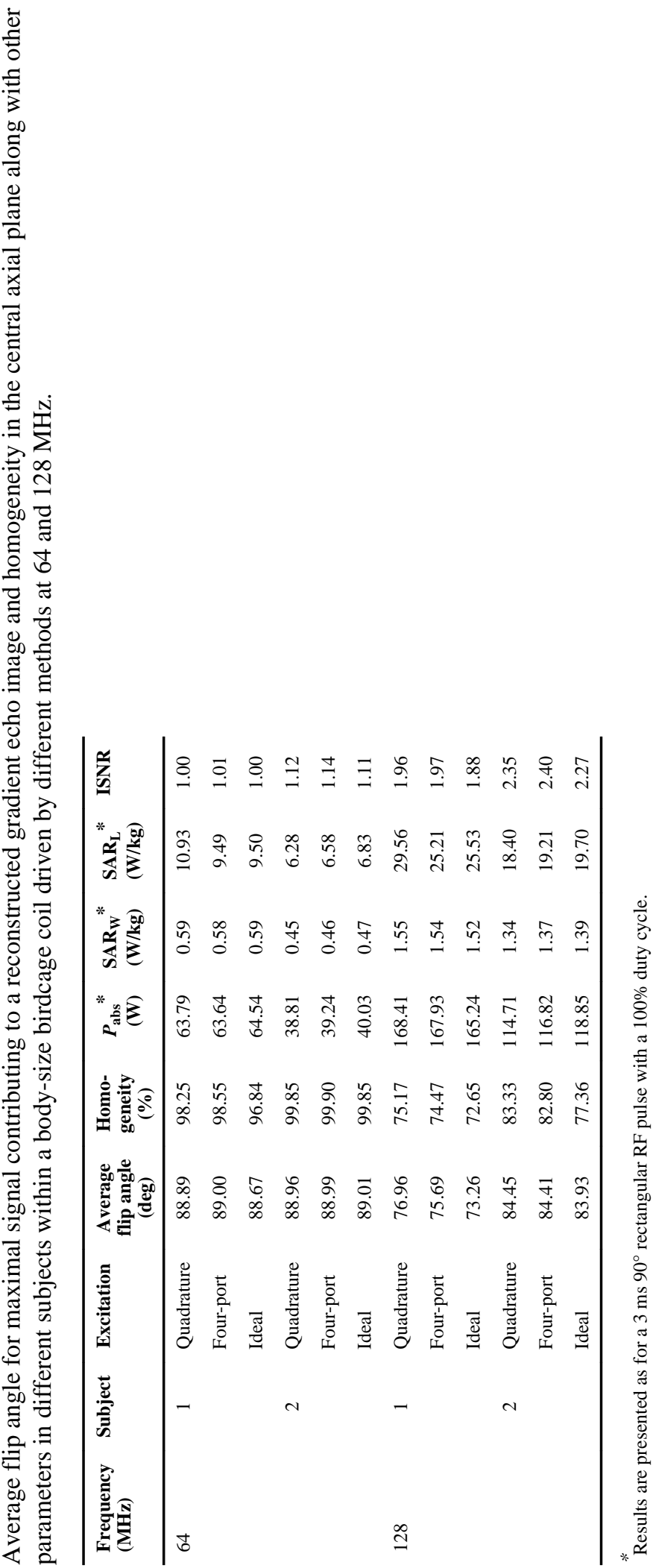

